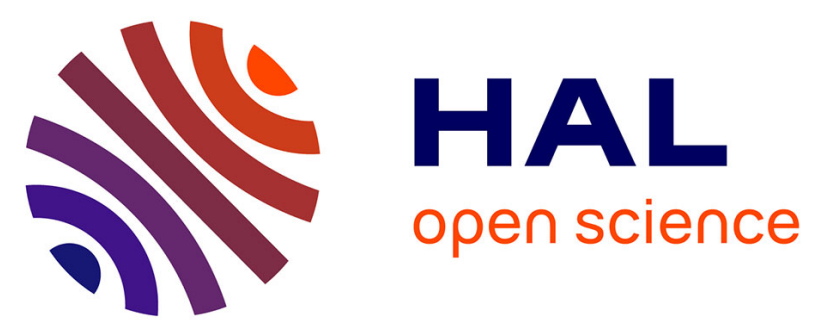

\title{
Prévention, pratiques médicales et gestion sanitaire au cours de la deuxième pandémie de peste
}

\author{
Sacha Kacki, Stefan Tzortzis, Dominique Castex, Michel Signoli
}

\section{To cite this version:}

Sacha Kacki, Stefan Tzortzis, Dominique Castex, Michel Signoli. Prévention, pratiques médicales et gestion sanitaire au cours de la deuxième pandémie de peste. Guy H.; Froment A. Archéologie de la santé, anthropologie du soin, La Découverte / Inrap, 2019, Archéologie de la santé, anthropologie du soin, 9782348045776. hal-02998369

\section{HAL Id: hal-02998369 \\ https://hal.science/hal-02998369}

Submitted on 11 Nov 2020

HAL is a multi-disciplinary open access archive for the deposit and dissemination of scientific research documents, whether they are published or not. The documents may come from teaching and research institutions in France or abroad, or from public or private research centers.
L'archive ouverte pluridisciplinaire HAL, est destinée au dépôt et à la diffusion de documents scientifiques de niveau recherche, publiés ou non, émanant des établissements d'enseignement et de recherche français ou étrangers, des laboratoires publics ou privés. 


\title{
Prévention, pratiques médicales et gestion sanitaire au cours de la deuxième pandémie de peste
}

\author{
S. Kacki*, S. Tzortzis**, D. Castex*, M. Signoli**
}

\author{
* PACEA, UMR 5199, Université de Bordeaux, France \\ ** ADÉS, UMR 7268, Faculté de Médecine, Marseille, France
}

Dans l'imaginaire collectif, la peste représente encore aujourd'hui, plus que toute autre maladie, le fléau par excellence. Le sinistre statut qu'on lui prête, loin d'être usurpé, trouve directement ses racines dans l'histoire passée des sociétés humaines, qui durent faire face durant des siècles à ses assauts répétés ${ }^{1}$. La maladie sévit notamment en Occident, où elle causa des épidémies récurrentes durant la majeure partie du Moyen Âge et de l'Époque moderne. Les populations d'alors, confrontées à une affection dont elles ignoraient tant la cause précise que le mode exact de propagation ${ }^{2}$, vécurent pendant des générations dans la crainte de ce mal inexorable. Pour autant, les communautés affectées ou menacées ne demeurèrent pas sans réaction face à la maladie; elles mirent progressivement en place des mesures individuelles et collectives de lutte contre la peste, visant tant à s'en prémunir qu'à soigner les malades et à gérer les conséquences sanitaires des épidémies.

La présente contribution, en s'intéressant au cas de la deuxième pandémie pesteuse (XIV $-\mathrm{XVIII}^{\mathrm{e}}$ siècles de notre ère) dans l'Ouest européen, vise à dresser un panorama des mesures ponctuelles puis généralisées qui, durant près de quatre siècles de flambées épidémiques plus ou moins localisées et espacées, ont été conçues et mises en œuvre par les sociétés occidentales pour faire face à la maladie. Si elle se fonde pour une large part sur l'exploitation de sources historiques, elle mobilise également certaines données fournies par l'archéologie, qui sont complémentaires des premières et livrent des informations inédites, notamment sur la gestion des cadavres durant ces crises de mortalité.

\footnotetext{
1 Si l'on sait aujourd'hui qu'une forme de peste affectait déjà les populations eurasiennes de l'âge du Bronze [Allentoft et al., ce volume], la maladie est surtout connue pour avoir entraîné trois grandes pandémies durant les périodes historiques - entre le $\mathrm{VI}^{\mathrm{e}}$ et le $\mathrm{VIII}^{\mathrm{e}}$ siècle de notre ère (première pandémie), entre le XIV siècle et le $\mathrm{XVIII}$ siècle (deuxième pandémie) et à partir de la fin du XIXe siècle (troisième pandémie).

2 La peste est causée par une bactérie (Yersinia pestis) qui affecte en premier lieu les rongeurs, mais peut être transmise à l'homme par l'intermédiaire de puces s'étant nourries sur des animaux infectés. L'hôte humain développe alors une infection caractérisée par l'éruption d'un ou plusieurs bubons, qui évolue le plus souvent vers une septicémie mortelle [Demeure et Carniel, 2009].
} 


\section{Théories médicales et croyances populaires}

Lorsque la maladie fit son retour en Europe au milieu du XIVe siècle, causant la tristement célèbre Peste noire (1347-1352), ses habitants n'avaient plus souvenir des grandes vagues de mortalité du haut Moyen Âge. La peste apparut donc comme une maladie nouvelle, dont on chercha dès lors à élucider la cause. Tout d'abord, les contemporains de l'épidémie furent quasiment unanimes à considérer ce mal comme l'expression du courroux de Dieu. Dans l'espoir d'en réchapper, les communautés menacées organisèrent fréquemment des processions religieuses. L'Église enjoignait par ailleurs ses fidèles à d'autres actes de dévotion, parmi lesquels des donations, des pèlerinages, ou encore, dans certaines villes d'Italie, la participation à des fraternités assurant le soin des malades et l'inhumation des victimes [Horrox, 1994].

Sans réfuter l'origine divine de la maladie, la médecine savante du XIV siècle avança d'autres thèses. La plupart des écrits médicaux de l'époque s'accordent ainsi sur le fait que la peste aurait eu pour cause première une corruption de l'air par des miasmes (théorie aériste). On reconnaissait à ces vapeurs toxiques plusieurs origines, incluant une mauvaise conjonction des planètes, de brusques changements du climat ou encore le vent du Sud [Weill-Parot, 2004 ; Dumas, 2003]. On considérait également que l'air vicié pouvait émaner des eaux stagnantes, des espaces confinés, ainsi que des excréments et des matières en putréfaction, au premier rang desquelles les cadavres [Biraben, 1976].

On avança enfin que la maladie pouvait être d'origine humaine. Des accusations furent portées contre toute personne extérieure aux communautés touchées, qu'il s'agisse d'étrangers, de voyageurs ou encore de lépreux. Mais, plus que tout autre, ce sont les juifs qui furent le plus fréquemment pointés du doigt; accusés de répandre la maladie en empoisonnant l'eau des puits, ils furent chassés, persécutés et parfois massacrés [Brossolet et Mollaret, 1994]. On peut, à cet égard, citer le cas de la ville espagnole de Tàrrega, où la fouille d'une partie du cimetière juif médiéval a révélé l'existence de plusieurs sépultures multiples ${ }^{3}{ }^{d u} x{ }^{e} v^{e}$ siècle constituées à la suite $d^{\prime}$ un pogrom [Colet et al., 2014].

Les concepts médicaux forgés durant la Peste noire ont prédominé tout au long de la deuxième pandémie. À partir de la fin du XV siècle, de nouvelles théories furent toutefois formulées quant à la nature et à la diffusion de la peste. La plus célèbre est sans nul doute celle du médecin Jérôme Fracastor, qui avança l'existence de germes spécifiques à la peste et défendit l'idée d'une contagion,

3 Sont qualifiées de multiples les sépultures renfermant les squelettes de plusieurs individus inhumés simultanément. 
pouvant selon lui s'opérer par voie aérienne, mais également par contact direct avec les malades ou leurs possessions [Fracastor, 1546]. Durant les siècles suivants, d'autres médecins soutinrent l'idée de la contagiosité de la peste, tel le père Athanase Kircher, qui l'attribua à des corpuscules animés et invisibles [Kircher, 1658]. Pour autant, la plupart des médecins restèrent farouchement opposés à ces théories contagionnistes. Le paroxysme de cette controverse fut atteint durant la grande peste de Marseille de 1720-1722. Si les partisans de la contagiosité étaient alors nombreux, ils se heurtèrent à l'avis de la quasi-totalité des membres de la faculté de médecine de Montpellier, au premier rang desquels Pierre Chirac, premier médecin du régent Philippe d'Orléans, qui ne voyait dans la maladie " qu'une fièvre maligne causée par les mauvaises nourritures du petit peuple de Marseille » [Bertrand, 1723, p. 110].

\section{Les mesures de prévention}

Empreintes des théories médicales et des croyances de leur époque, les communautés menacées par la peste tentèrent au mieux de s'en prémunir, recourant pour cela à différentes attitudes et à divers artifices. En premier lieu, la solution retenue par certains fut de prendre la fuite. Cette posture, adoptée par les nobles et les bourgeois fortunés, qui disposaient de ressources suffisantes pour subsister en un autre lieu, s'étendit parfois aux franges plus modestes de la population. Prendre la fuite fut sûrement salvateur pour certains; mais cette attitude contribua aussi à disséminer la maladie, pour peu que certains fuyards fussent contaminés.

Pour ceux qui ne pouvaient fuir, la prévention passa principalement par le recours à diverses substances odoriférantes, utilisées comme rempart contre l'air vicié. Il était préconisé de brûler dans les maisons des épices et aromates (troncs de choux, pelures de coings) ou certaines essences de bois (genièvre) et de se munir d'un bouquet d'herbes aromatiques quand on sortait. On recourait également à des amulettes anti-pesteuses (médailles à l'effigie de certains saints, talismans faits de matériaux réputés prophylactiques), dont certains exemplaires ont été découverts en contexte archéologique (fig. 1). L'air corrompu pouvant aussi s'insinuer par les pores de la peau, il était conseillé d'éviter les activités qui les dilatent (bains, relations sexuelles, consommation excessive de nourriture et de boissons alcoolisées). Les médecins préconisaient enfin certains traitements médicaux traditionnels telles les saignées et les purges.

Constatant la faible efficacité de ces moyens de protection individuels, les pouvoirs politiques prirent progressivement des mesures collectives contre la peste. Dès la fin du XIV siècle, les autorités 
vénitiennes nommèrent des officiers civils pour édicter des règlements préventifs [Biraben, 1976]. En France, de nombreuses villes se dotèrent dès le $x v^{e}$ siècle d'un " bureau de santé ", dont le personnel était chargé de nettoyer les rues, d'abattre les animaux errants, de marquer les maisons infectées et de désinfecter les possessions des malades. Mais par-dessus tout, la principale mesure fut l'isolement systématique des personnes infectées ou suspectées de l'être. Les mises en quarantaine, adoptées ponctuellement jusqu'alors, comme à Raguse [Grmek, 1959] ou à Venise [Panzac, 1986], se généralisèrent peu à peu pour devenir la règle absolue dans le courant du XVI ${ }^{\mathrm{e}}$ siècle [Biraben, 1976]. De façon concomitante, les bureaux de santé exigèrent des « billets de santé » pour entrer dans les villes. Cet usage s'étendait au domaine maritime où ces certificats prenaient le nom de "patentes", dites « nettes " si les bateaux venaient d'un port non contaminé, " brutes » s'ils arrivaient d'un pays infecté. Dans le second cas, le navire devait faire quarantaine dans un lazaret, où passagers et équipage étaient placés en observation. Les cargaisons y étaient dans le même temps déballées pour être exposées à l'action purificatrice de l'air [Brossolet et Mollaret, 1994].

L'histoire et le fonctionnement des lazarets sont relativement bien connus par les sources textuelles [Panzac, 1986]. Le cas de Marseille est particulièrement emblématique. La cité, que son rôle éminent dans les échanges commerciaux avec le Levant et l'Afrique du Nord exposait fortement au péril épidémique, se dota d'un lazaret dès 1526. Déménagé à deux reprises aux XVI ${ }^{\mathrm{e}}$ et $\mathrm{XVII}$ siècles pour finalement être implanté près de l'anse d'Arenc, cet établissement accueillait les passagers et cargaisons, tandis que les navires faisaient quarantaine sur une île au large de la ville. Un intendant de santé inspectait la mise en purge des cargaisons (étalage et exposition à l'air), autorisait ou refusait l'entrée des navires et déterminait la durée d'isolement des personnes et des marchandises. Les secondes étant considérées comme principal vecteur de la maladie, elles pouvaient être conservées sur de longues périodes, allant de trente à quarante jours pour les patentes nettes et jusqu'à soixante jours pour les patentes brutes ${ }^{4}$. Dans ces conditions, le lazaret devint rapidement encombré. Son emprise fut donc augmentée une première fois après la peste de 1720 (création de l'enclos neuf des marchandises et de son port), puis une seconde fois à la fin du XVIII siècle.

Si le lazaret d'Arenc couvrait dans son dernier état une surface de près de 25 hectares, aucun de ses édifices et équipements n'est aujourd'hui conservé dans la topographie de la ville. On dispose toutefois, depuis peu, de données archéologiques le concernant. Plusieurs diagnostics archéologiques réalisés entre 2003 et 2015 ont en effet permis d'en détecter les vestiges. Une fouille a en outre eu lieu en 2012 sur une partie de l'enclos neuf des marchandises. Cette opération a mis au

4 Cette durée variait également selon le type de marchandises. Elle était par exemple plus longue pour les matières textiles, le cuir ou les fourrures, plus courte pour les denrées alimentaires. 
jour les piliers de l'une des halles, plusieurs bâtiments, ainsi que les calades servant au déballage des textiles [Newman et al., 2013].

En parallèle de la généralisation des quarantaines et de l'emploi des lazarets, d'autres mesures draconiennes, édictées cette fois à l'échelle des États, virent le jour dans le courant du XVII ${ }^{\mathrm{e}}$ siècle. On mit ainsi en place, durant les épidémies, des cordons sanitaires qui assuraient l'isolement des localités touchées et rendaient impossible la fuite de leurs habitants. Par ailleurs, un réseau international d'informations sanitaires s'organisa progressivement, permettant de conjuguer les efforts de différents pays pour lutter contre la diffusion de la peste [Biraben, 1976]. Ces nouvelles mesures, bien plus efficientes que celles adoptées durant les siècles précédents, permirent finalement de repousser la peste hors d'Europe occidentale dans le courant du XVIII siècle $^{5}$.

\section{La prise en charge des malades}

En l'absence de mesures de prévention efficaces durant la majeure partie de la deuxième pandémie, la maladie causa, au gré de vagues épidémiques récurrentes, la contamination de très nombreux individus que leurs contemporains durent assister et tentèrent de soigner. Les mesures adoptées pour cela furent naturellement étroitement liées aux concepts d'alors concernant les causes et les modes de diffusion de l'affection. Pour les médecins de la fin du Moyen Âge, convaincus que la peste était causée par des miasmes charriés par l'air, nul n'était besoin d'éloigner les malades de leur entourage encore sain. Tous étaient donc assignés à résidence, ce qui conduisait le plus souvent à la contamination de l'ensemble de la maisonnée [Brossollet et Mollaret, 1994]. Dans les siècles suivants, les partisans de la théorie contagionniste obtinrent l'adoption de réelles mesures $\mathrm{d}^{\prime}$ isolement. À partir de la seconde moitié $\mathrm{du} x \mathrm{xl} \mathrm{I}^{\mathrm{e}}$ siècle, il devint ainsi d'usage de reléguer les malades dans des structures dédiées, à l'écart des lieux d'habitation [Biraben, 1976]. Ces infirmeries de peste, généralement constituées de baraquements en bois, étaient édifiées par le personnel du bureau de santé et brûlées sitôt après la fin de l'épidémie.

Les recherches archéologiques menées en France ont permis de réaliser des fouilles à l'emplacement de deux infirmeries de peste, situées respectivement à Lambesc dans les Bouches-du-Rhône [Bizot et al., 2005] et à Puy-Saint-Pierre dans les Hautes-Alpes [Signoli et al., 2007]. Pour le premier de ces sites, les archéologues disposaient d'une riche documentation textuelle désignant le terrain exploré

5 D'autres causes au recul de la peste ont toutefois été avancées et pourraient avoir joué un rôle concomitant, qu'elles soient d'ordre écologique (remplacement des espèces murines en présence) ou microbiologique (déclin de virulence ou de transmissibilité des souches bactériennes) [Brossollet et Mollaret, 1994]. 
comme l'emplacement d'une infirmerie de peste utilisée durant le printemps et l'été 1590. Aucun vestige des cabanes de cette structure de soin n'a toutefois été mis au jour lors de la fouille, seul subsistant le cimetière attenant. Le second site, en relation avec une épidémie qui frappa la région de Briançon en 1629-1630, était encore, au moment de sa découverte, signalé par une croix marquant l'emplacement du cimetière. Là encore, seuls des vestiges funéraires ont pu être identifiés.

Les soins médicaux apportés aux pestiférés furent peu diversifiés. Ils consistaient, d'une part, à l'administration de différents mélanges de plantes aromatiques - notamment la thériaque ${ }^{6}-$ et, d'autre part, à l'incision des bubons. Cette opération, effectuée par des barbiers-chirurgiens, fut pratiquée dès la Peste noire ; Guy de Chauliac (1298-1368) préconisait ainsi d'apposer un emplâtre de figues et d'oignons cuits sur le bubon pour le faire mûrir, avant de l'ouvrir pour en extraire le poison, puis de cautériser la plaie [Nicaise, 1890]. Ces méthodes de soin restèrent en vigueur durant toute la deuxième pandémie, seules évoluant les précautions prises par les médecins pour se prémunir d'être contaminés. En parallèle de l'émergence des théories contagionnistes, on inventa des bistouris et des cautères à longs manches pour opérer les bubons à relative distance des malades [Brossollet et Mollaret, 1994]. Les médecins se dotèrent aussi d'artifices assurant une séparation physique d'avec ces derniers. C'est ainsi qu'apparut au début du XVII ${ }^{\mathrm{e}}$ siècle le costume inventé par Charles de Lormes, qui couvrait la quasi-totalité du corps, tout en disposant d'un nez empli de substances odoriférantes. Les fervents défenseurs de la théorie aériste n'hésitaient toutefois pas à approcher les pestiférés, voire à autopsier leurs cadavres. Un exemple archéologique l'atteste : la fosse sépulcrale du site marseillais de l'Observance, contemporaine de l'épidémie de 1722, a livré un squelette d'adolescent dont la boîte crânienne avait été ouverte par sciage, selon une procédure en tous points similaire à celle décrite dans les ouvrages de chirurgie de l'époque [Signoli et al., 1997].

\section{L'inhumation des cadavres de pestiférés: entre traditions funéraires et gestion sanitaire}

Dans un contexte où la prophylaxie et les soins demeurèrent longtemps inefficaces, la peste provoqua des décès innombrables. L'augmentation subite du nombre de défunts sur un court laps de temps constitua naturellement un problème de santé publique, d'autant plus exacerbé que les corps en putréfaction furent longtemps considérés comme l'une des sources des miasmes. Les populations touchées durent donc mettre en œuvre des moyens adaptés pour gérer cet afflux de cadavres. C'est

6 Cette préparation, composée de plusieurs dizaines d'ingrédients, avait la réputation de guérir toutes les maladies. 
indéniablement sur cet aspect que l'archéologie apporte la plus importante contribution, de nombreux cimetières de pestiférés ayant fait l'objet d'opérations de fouilles (fig. 2). Le lien entre ces sites et la maladie est attesté, parfois par des sources écrites, le plus souvent par des analyses paléomicrobiologiques ayant identifié l'ADN de Yersinia pestis dans des restes squelettiques.

Les découvertes archéologiques démontrent, en premier lieu, le recours fréquent à l'inhumation simultanée de plusieurs corps dans une même fosse sépulcrale. Si l'abandon ponctuel de l'inhumation individuelle $^{7}$ constitue certes une entorse aux usages funéraires traditionnels, les sépultures de pestiférés du XIV siècle rendent toutefois compte de la volonté des populations de respecter les pratiques d'usage en ce qui concerne le positionnement des corps. À de rares exceptions près, les individus reposant dans ces tombes étaient en effet déposés sur le dos, selon une même orientation est/ouest, à l'instar de la plupart des défunts inhumés dans les cimetières médiévaux [Kacki et Castex, 2012]. Le soin porté aux victimes de la Peste noire transparaît également dans leur lieu d'inhumation, à savoir, dans la majorité des cas, le cimetière paroissial.

Dans les siècles suivants, la gestion funéraire évolua de manière sensible. Parallèlement aux mesures $d^{\prime}$ isolement des malades, les interdictions d'inhumer les cadavres en ville se multiplièrent et les municipalités acquirent des terrains hors leurs murs pour enterrer les morts de la peste. La plupart des sites d'inhumation de pestiférés postérieurs au XIV ${ }^{e}$ siècle correspondent ainsi à des créations ex nihilo, à l'exemple des cimetières d'infirmeries de peste précédemment mentionnés des Fédons et de Lariey. Les données archéologiques rendent également compte d'une modification des pratiques encadrant la mise en terre, la standardisation dont témoignaient les tombes du XIV siècle quant au positionnement des cadavres s'effaçant peu à peu. Pour les sites des $\mathrm{XV}^{\mathrm{e}}$ et $\mathrm{XVI} \mathrm{I}^{\mathrm{e}}$ siècles, l'orientation des corps est plus hétérogène, les dispositions tête-bêche et les positions atypiques plus fréquentes. Il semble ainsi que la rationalisation de l'espace d'inhumation et les efforts pour limiter les contacts avec les cadavres ont progressivement pris le pas sur le respect des normes chrétiennes [Castex et Kacki, 2015].

Les modifications amorcées aux $X V^{e}$ et $x V l^{e}$ siècles ne firent que s'amplifier dans les siècles suivants, les pouvoirs politiques imposant progressivement un cadre plus strict à l'ensevelissement des cadavres de pestiférés. L'épidémie que connut Marseille et une partie de la Provence entre 1720 et 1722 constitue un exemple emblématique de ces dispositions, documentées par les sources écrites,

7 Les sépultures multiples, si elles constituent les témoins archéologiques les plus patents des phénomènes épidémiques anciens, ne furent toutefois pas adoptées de manière systématique. Elles côtoient ainsi, dans de nombreux sites d'inhumation de pestiférés, des sépultures individuelles de victimes de la maladie [Bizot et al., 2005 ; Kacki et al., 2011]. 
mais également par plusieurs découvertes archéologiques effectuées à Marseille et dans la proche ville de Martigues.

À Marseille, la flambée épidémique fut telle que les cadavres s'accumulèrent dans les rues et dans les structures de soin. Pour pallier cette situation, il fallut multiplier les creusements de tombes, au prix d'une mobilisation sans précédent et sous la contrainte de paysans et de centaines de forçats. La gestion de l'épidémie aurait finalement nécessité l'aménagement d'au moins vingt-sept grandes fosses à inhumation, creusées principalement aux abords du rempart et des portes de la ville, mais aussi localement dans son périmètre intra-muros. Ce dernier cas de figure s'applique aux deux structures funéraires qui ont à ce jour fait l'objet de fouilles archéologiques. La première d'entre elles, localisée dans le jardin du couvent de l'Observance, correspondait à une vaste structure quadrangulaire. Elle a livré des squelettes reposant selon des attitudes et des orientations variées, qui étaient concentrés sur une seule de ses extrémités. Cette configuration singulière suggère que le creusement était destiné à accueillir un plus grand nombre de cadavres. Cela s'explique probablement par le fait que la fosse est contemporaine de la rechute épidémique de 1722 , témoignant ainsi d'un souci de prévoyance en remémoration de la principale flambée épidémique intervenue quelques mois plus tôt [Dutour et al., 1994]. La seconde structure, située à proximité de la cathédrale de la Major, correspondait à une fosse oblongue, malheureusement très largement tronquée et écrêtée par des aménagements postérieurs. Cette excavation, contemporaine de la flambée épidémique de 1720, était densément comblée de squelettes sur toute sa surface. Les corps y reposaient sans position ni orientation préférentielle et étaient associés à des éléments vestimentaires et mobiliers [Tzortzis et al., 2010], attestant que les individus n'avaient pas été dépouillés de leurs effets personnels.

À Martigues, l'épidémie aurait été plus facilement maîtrisée en matière de gestion funéraire. Les deux sites d'inhumation de pestiférés qui y ont été mis au jour sont localisés l'un et l'autre hors les murs de la ville. Le premier de ces sites (le Délos), en grande partie détruit, n'a pu faire l'objet que d'une fouille d'urgence. Trois tranchées parallèles de largeur et de profondeur relativement modestes, renfermant un nombre important de squelettes aux postures diverses, y ont été mises au jour [Signoli, 2006]. Pour le second site (les Capucins de Ferrières), la découverte est intervenue suffisamment tôt pour permettre une fouille quasi-exhaustive. La fouille des cinq tranchées parallèles qu'il a livrées a révélé certaines nuances dans les modes opératoires de dépôt des corps [Tzortzis et Signoli, 2009]. Ainsi, la disposition des squelettes dans les tranchées témoigne, selon les cas, de déchargements directs et successifs de tombereaux de cadavres ou de dépôts manuels, effectués selon des procédés variables (fig. 3). Par ailleurs, une des tranchées se distinguait des 
quatre autres par une moindre cadence des inhumations et le dépouillement des cadavres de leurs vêtements, ce qui suggère que les défunts ne venaient pas des convois mortuaires arrivant de la ville, mais plutôt d'une infirmerie voisine occupant le couvent des Capucins.

II convient enfin de mentionner l'usage fréquent de chaux dans les sépultures de la fin de la deuxième pandémie. Documenté dans la plupart des tombes de pestiférés des $\mathrm{XVII}$ et $\mathrm{XVIII}$ siècles fouillées à ce jour, son emploi est au contraire rarissime pour les sites plus anciens [Kacki et Castex, 2014]. Cette évolution accompagne les préconisations répétées des pouvoirs politiques, qui recommandèrent à partir du XVII ${ }^{e}$ siècle l'usage de ce matériau pour accélérer la décomposition des corps et limiter les odeurs.

In fine, l'archéologie révèle divers aspects de la gestion funéraire, dont certains ne sont que peu, voire pas, abordés par les sources textuelles. Ces données sont de première importance pour retracer l'évolution dans le temps des modalités d'inhumation des cadavres de pestiférés (fig. 4) et pour appréhender la diversité des pratiques adoptées lors d'une même épidémie.

\section{Conclusion}

Si elles ne parvinrent jamais à élucider la cause de la peste, les populations de la fin du Moyen Âge et de l'Époque moderne déployèrent de multiples efforts pour tenter de la combattre et d'en maîtriser les conséquences sanitaires. Les mesures qu'elles adoptèrent ont connu une évolution au fil des siècles, à la faveur d'observations empiriques, de l'émergence de nouvelles théories médicales et d'une implication grandissante des pouvoirs politiques dans la gestion des épidémies. Ces modifications, que permettent d'appréhender les sources écrites, mais également certains vestiges archéologiques, ont peu à peu conduit au développement de véritables politiques de santé publique en Europe. Certes fondées sur des procédés d'efficacité inégale, elles n'en demeurent pas moins une préfiguration de celles qui ont cours aujourd'hui. Car loin de ne représenter qu'une préoccupation des populations anciennes, la prévention et la lutte contre les maladies émergentes et réémergentes constituent encore de nos jours de réels enjeux de société, qu'exacerbe d'autant plus I'apparition de souches pathogènes résistantes aux antibiotiques. 


\section{Références bibliographiques}

BERTRAND J.-B. (1723), Relation historique de tout ce qui s'est passé à Marseille pendant la dernière peste. Seconde édition corrigée et augmentée, Cologne, Pierre Marteau.

BIRABEN J.-N. (1976), Les Hommes et la peste en France et dans les pays européens et méditerranéens, Tome II : Les Hommes face à la peste, Paris-La Haye, Mouton.

Bızot B. et al. (2005), La saison d'une peste (avril-septembre 1590). Le cimetière des Fédons à Lambesc, Paris, CNRS éditions, $131 \mathrm{p}$.

Brossollet J. et Mollaret H. H. (1994), Pourquoi la peste ? Le rat, la puce et le bubon, Paris, Gallimard (collection Découvertes, $\mathrm{n}^{\circ} 229$, série Sciences et techniques), $160 \mathrm{p}$.

CASTEX D. et KACKI S. (2015), "L'impact des épidémies sur les usages funéraires du passé. Faits archéologiques versus idées reçues ", in TREFFORT C. (dir.), Le Cimetière au village dans l'Europe médiévale et moderne, Actes des XXXVe journées internationales d'histoire de Flaran, Toulouse, Presses universitaires du Midi, p. 233-251.

ColEt A. et al. (2014), "The Black Death and Its Consequences for the Jewish Community in Tàrrega: Lessons from History and Archeology ", The Medieval Globe, vol. 1, p. 63-96.

Demeure C. et Carniel E. (2009), "Yersinia pestis ", Revue Francophone des Laboratoires, vol. 415, p. 33-39.

DUMAS G. (2003), "La fenêtre dans les traités de peste de la région de Montpellier aux XIV et $\mathrm{XV}^{\mathrm{e}}$ siècles ", in ConNoChIE-BOURGNe C. (dir.), Par la fenestre : Études de littérature et de civilisation médiévales, Aix-en-Provence, Presses universitaires de Provence, p. 157-165.

Dutour O. et al. (1994), « Le charnier de la Grande Peste de Marseille (rue Leca) : données de la fouille de la partie centrale et premiers résultats anthropologiques ", Préhistoire et Anthropologie méditerranéennes, vol. 3, p. 191-203.

FRACASTOR J. (1546), De sympathia et antipathie rerum liber unus, de contagione et contagiosis morbis et curative libri III, Venise, Luntae.

GRMEK M. D. (1959), « Quarantaine à Dubrovnik », Symposium Ciba, vol. 7, p. 30-33.

HORROX R. (1994), The Black Death, Manchester, Manchester University Press, 364 p.

KACKI S. et CASTEX D. (2012), « Réflexions sur la variété des modalités funéraires en temps d'épidémie. L'exemple de la Peste noire en contextes urbain et rural ", Archéologie médiévale, vol. 42, p. 1-21.

KACKI S. et CASTEX D. (2014), « La sépulture multiple de la basilique des saints martyrs Just et Pastor : bioarchéologie des restes humains ", Quaderns d'Arqueologia i Història de la Ciutat de Barcelona, vol. 10, p. 180-199. 
KACKI S. et al. (2011), « Black Death in the rural cemetery of Saint-Laurent-de-la-Cabrerisse (AudeLanguedoc, southern France, 14th century): immunological evidence ", Journal of Archaeological Science, vol. 38, p. 581-587.

KIRCHER A. (1658), Scrutinium physico-medicum contagiosae luis quae pestis dicitur, Leipzig, Bauerianis.

Newman C., Michel J. et Touma M. (2013), " Marseille, 104 boulevard de Paris », Bilan scientifique régional PACA 2012, DRAC, Service régional de l'Archéologie, p. 137-138.

NICAISE E. (1890), La Grande Chirurgie de Guy de Chauliac, chirurgien, maître en médecine de I'université de Montpellier, composée en l'an 1363, Paris, Alcan.

PANZAC D. (1986), Quarantaines et lazarets: I'Europe et la peste d'Orient (XVII'-XX siècles), Aix-enProvence, Édisud.

SIGNOLI M. (2006), Étude anthropologique de crises démographiques en contexte épidémique : aspects paléo et biodémographiques de la peste en Provence, Oxford, Archaeopress (British Archaeological Reports, International Series, vol. 1515).

SIGNOLI M. et al. (1997), " Mise en évidence d'une autopsie crânienne réalisée pendant la Grande Peste de Marseille (1720-1722) ", Comptes rendus de l'Académie de Sciences, vol. 320 (7), p. 575580.

SIGNOLI M. et al. (2007), " Découverte d'un cimetière de pestiférés du XVII siècle (Puy-Saint-Pierre, Hautes-Alpes, France) ", in SIGNOLI M. et al. (dir.), Peste : entre épidémies et sociétés, Florence, Firenze University Press, p. 97-103.

TZORTZIS S. et al. (2010), « Archives historiques et archives biologiques de la peste : intérêts du passé et enjeux actuels ", in Delestre X. et MARCHESI H. (dir.), Archéologie des rivages méditerranéens : 50 ans de recherche, Paris, Éditions Errance / Ministère de la Culture et de la Communication, p. $449-460$.

Tzortzis S. et Signoli M. (2009), « Les tranchées des Capucins de Ferrières (Martigues, Bouches-duRhône, France). Un charnier de l'épidémie de peste de 1720 à 1722 en Provence ", Comptes rendus Palevol, vol. 8, n. 8, p. 749-760.

WeILL-PAROt N. (2004), "La rationalité médicale à l'épreuve de la peste : médecine, astrologie et magie (1348-1500) ", Médiévales, vol. 46, p. 73-88. 


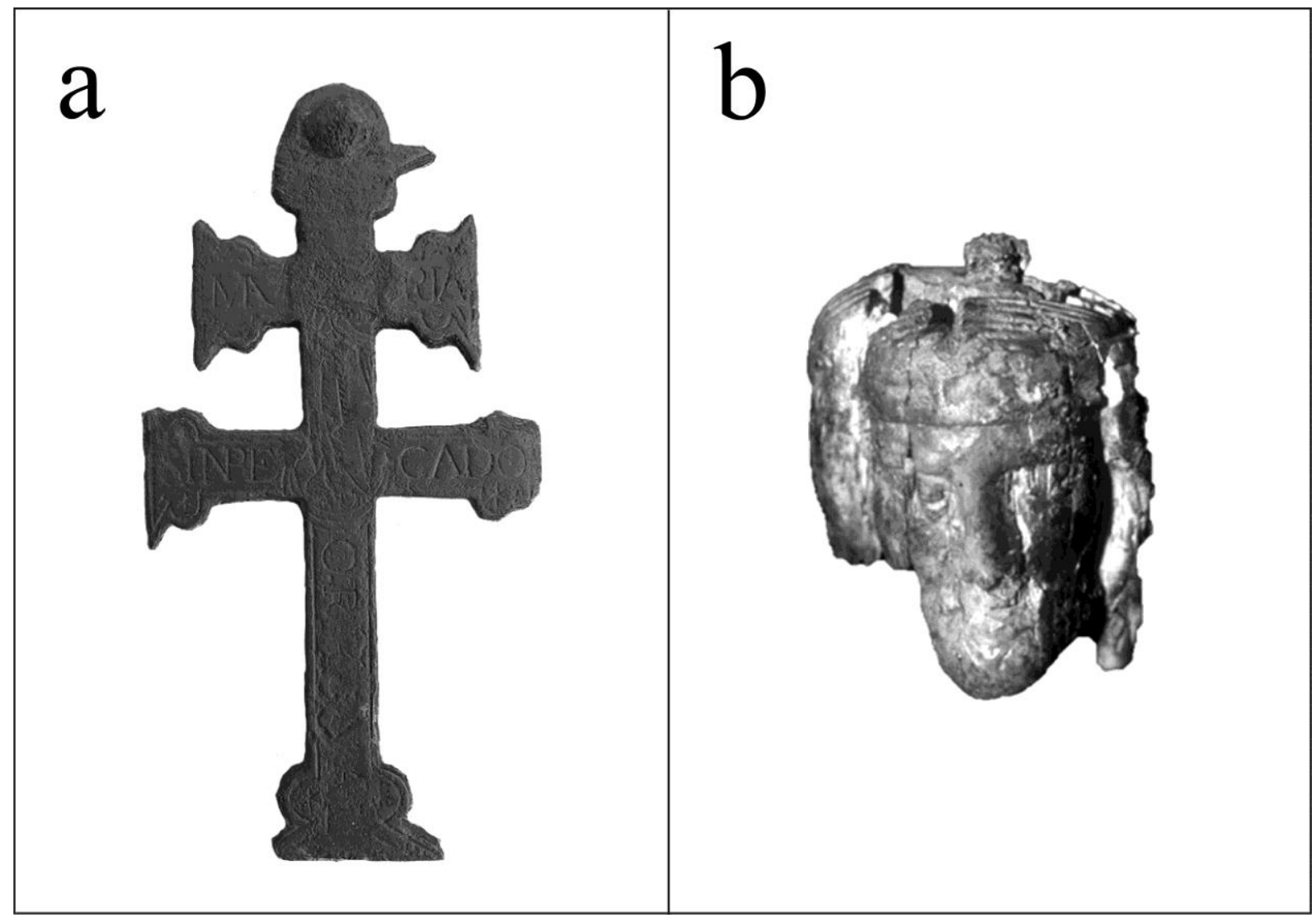

Figure 1. Croix de peste en alliage cuivreux (a) et pendentif en buis sculpté en forme de tête de Christ (b) mis au jour dans le cimetière de pestiférés du Délos (Marseille, 1720-1721) (clichés S. Tzortzis [a] et M. Signoli [b]).

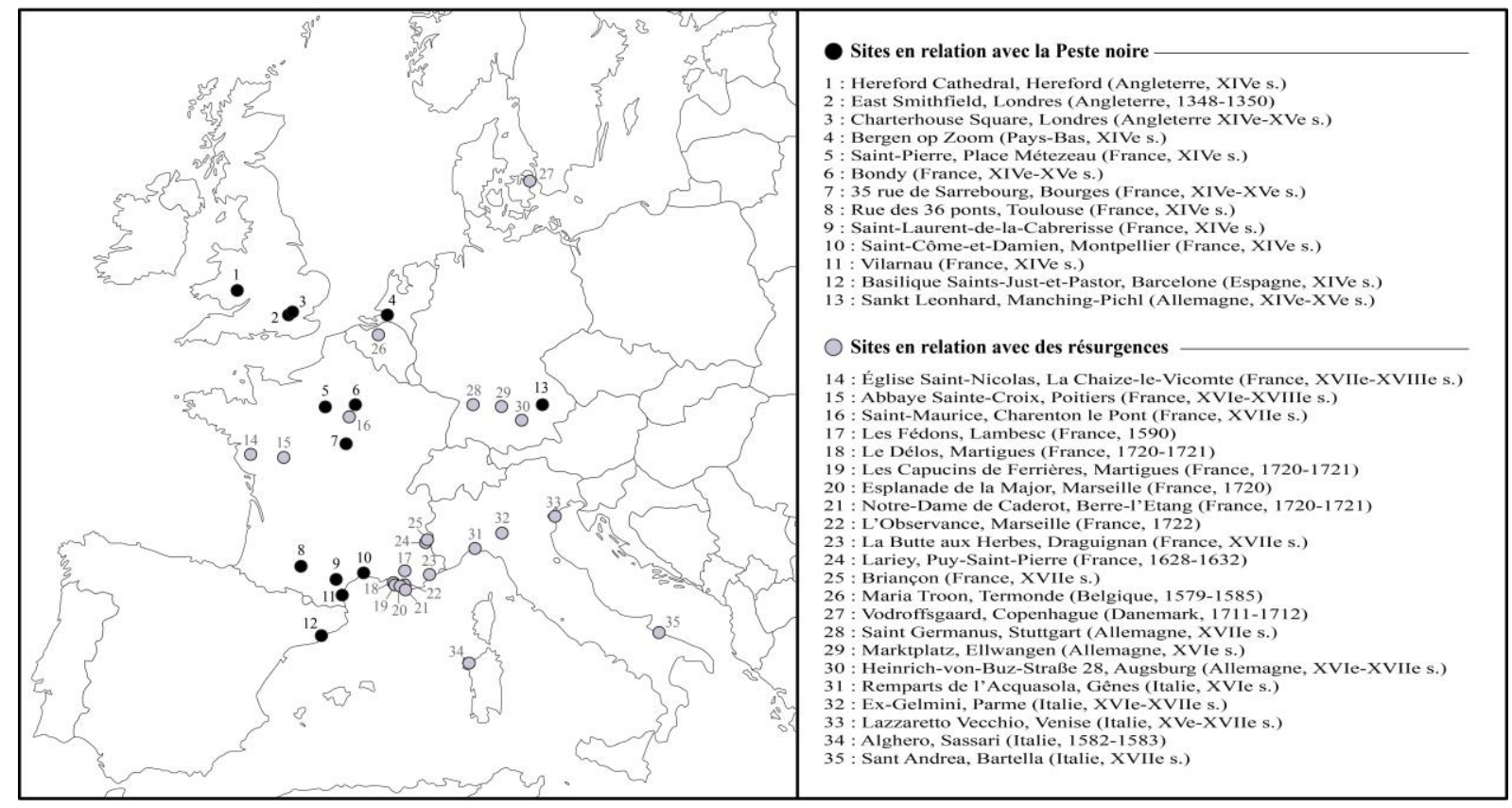

Figure 2. Carte de répartition des sites funéraires européens en relation avec la deuxième pandémie de peste (DAO S. Kacki). 

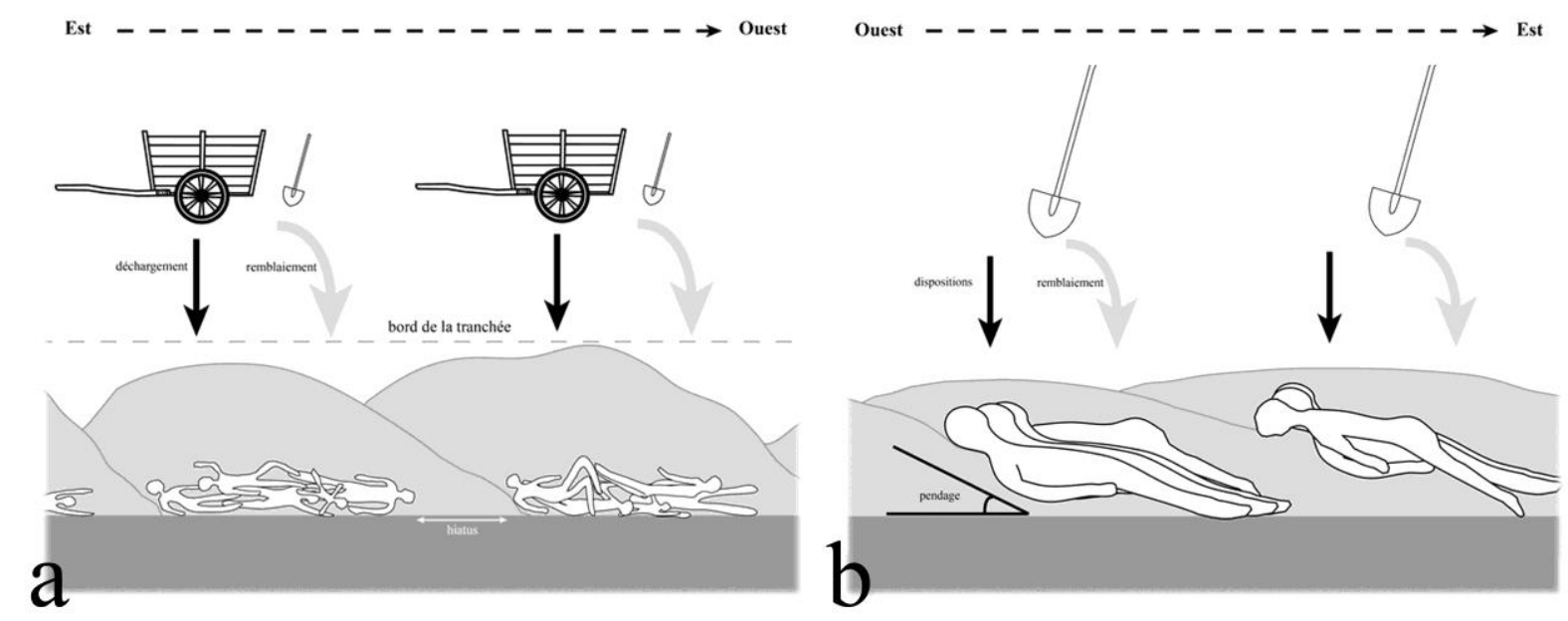

Figure 3. Restitution du mode opératoire de dépôts des cadavres dans la tranchée I (a) et la tranchée IV (b) du site des Capucins de Ferrières (DAO S. Tzortzis).

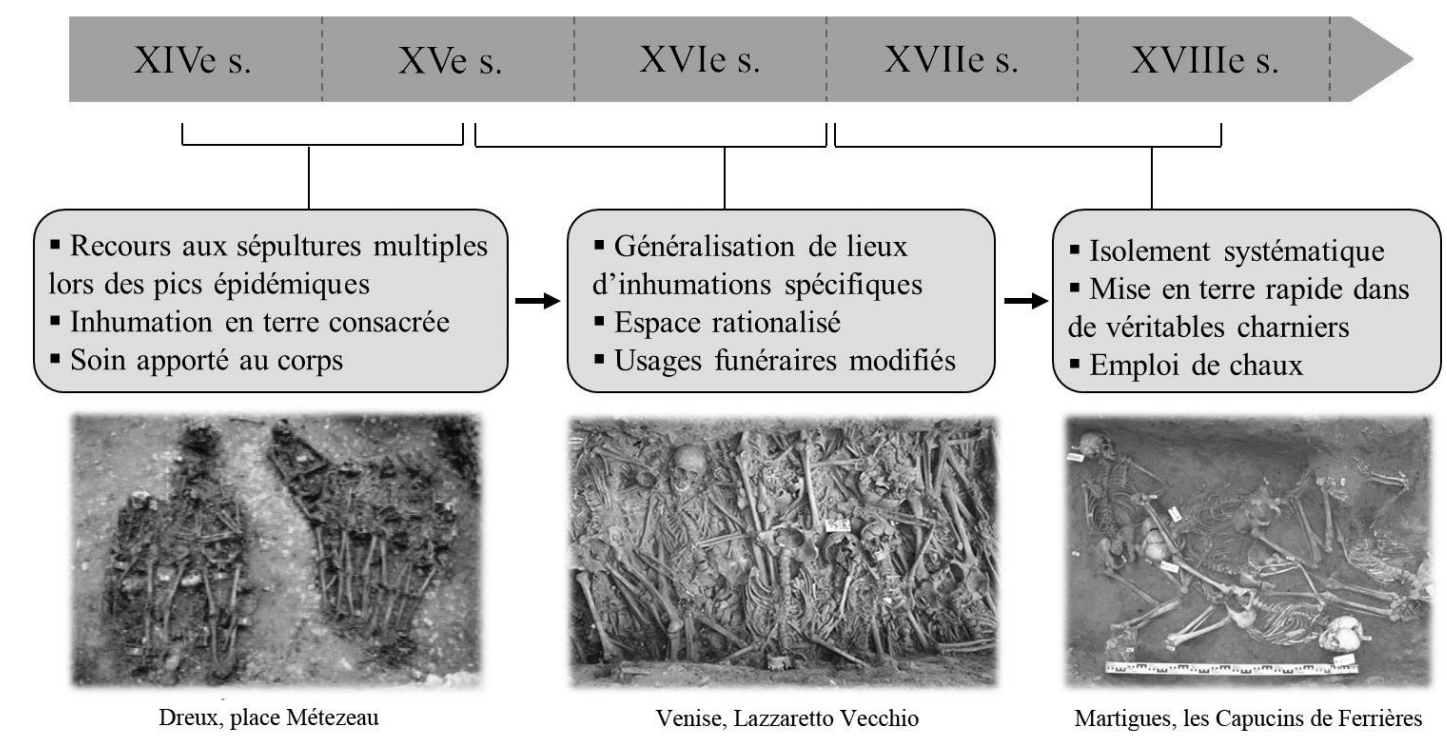

Figure 4. Bilan synthétique de l'évolution des modalités d'inhumation des cadavres durant les épidémies de peste contemporaines de la deuxième pandémie (DAO S. Kacki). 